\title{
THE CONSTRUCTION FEATURES OF TRADITIONAL SETTLEMENTS IN THE MIDDLE AREA OF JIANGXI PROVINCE BASING ON THE ANALYSIS OF TANGYIN TOWN
}

\author{
JUANJUAN FU ${ }^{\mathrm{a}}$ \\ ${ }^{\text {a }}$ Heritage Planning Department, Tsinghua Heritage Institute for Digitization, Shangdi West Road, Haidian District, Beijing, China- \\ fujuanjuan@thid.cn
}

KEY WORDS: Traditional Settlement, Construction Features, Formation Logic, Tangyin Town, Middle of Jiangxi Area,

\begin{abstract}
:
Based on general investigations and utilizing GIS software, missing historical data was hypothesized. From the perspectives of social and economic development, this paper tries to find the logic behind the formation and development of traditional settlements. Using systematic examination, the features of the town's overall layout, courtyard shape and building materials appear. Applying comparative analysis, this paper indicated the similarities and differences between Tangyin town and the other areas of Jiangxi, and add them to the construction logic of the middle area in Jiangxi to make the following construction activities much in order.
\end{abstract}

\section{INTRODUCTION}

The traditional settlement researches in Jiangxi province are mainly on the following facets ${ }^{[1]}$ : (1) The combination with the society and philosophy;(2) The combination of the form and the environment;(3) The combination of the construction and the design method;(4) The combination of the conservation, the transformation and the development. But all these researches are almost based on one point. This paper is based on one typical case to connect all the factors in series which will make the development history and the influencing mechanism more clearly.

Tangyin is a historic and cultural town in Fuzhou city of Jiangxi province, which has a history of over 970 years. Accompanied by rapid urbanization and original residents abroad, the vernacular dwellings are facing demolition or the risk of collapse, which need the emergency reinforcement in urgent.

Through the census, the paper clarified the existing number of vernacular dwellings, the courtyard types, the building structure, the building decoration and etc. By comparison in the background of the middle area in Jiangxi, the characteristics and formation mechanism of the traditional settlements had been extracted, which may direct for the subsequent architectural evaluation, the protection nomination and etc.

\section{GENERAL LAYOUT}

\subsection{The internal momentum of the development ${ }^{[2]}$}

Tangyin was famous for grass cloth, which was one of the two famous textile industry cores in Jiangxi, basing on the peculiar water which could make the grass cloth more whiter. Because of this background, Tangyin became to be one of the famous grass cloth production and distribution center to late Ming and early Qing dynasties. Numerous merchants had been here to trade with the aid of Yi river which promoted the construction of the wharfs. On the other side, more and more rich businessmen returned hometown and constructed dwellings and ancestral halls .The settlement gradually formed.
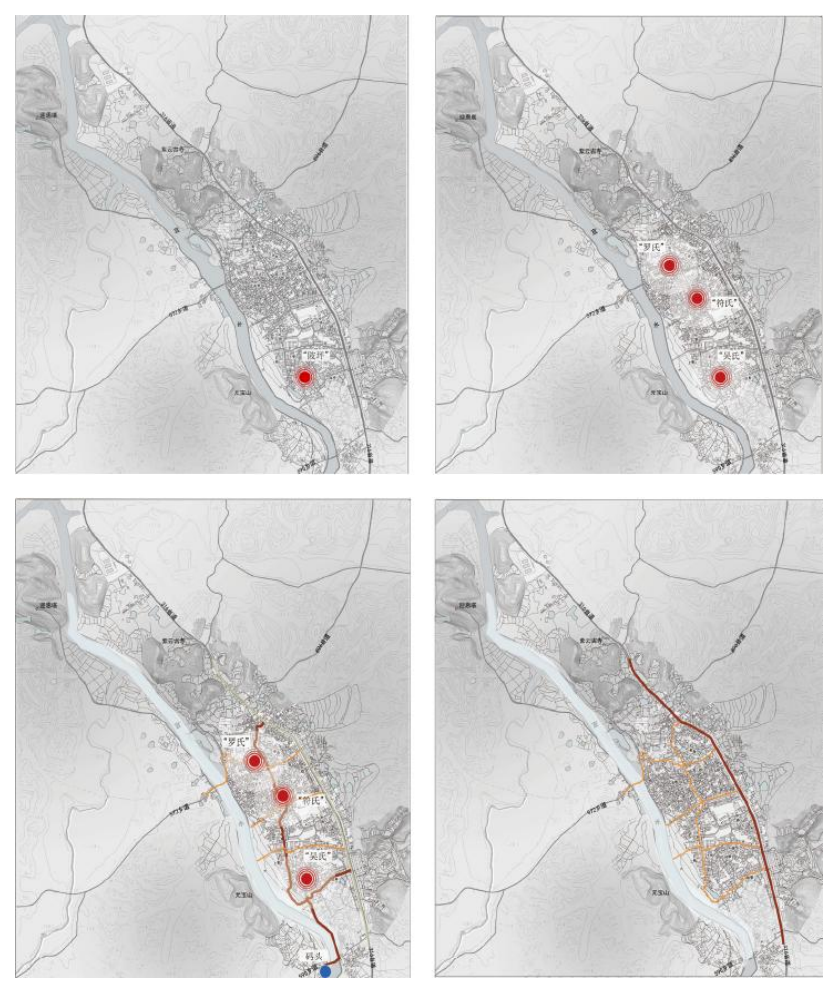

Figure 1. The development phases of Tangyin town

\subsection{The natural appearance of the basement}

According to fengshui, the residents built dwellings with well implied meaning. Predecessors constructed in their selected address point successively, which formed to be "he lu". According to the distance between "he lu", local residents then divided "he lu" into "eleven fang" from north to south, including "shujin fang", "zhoujin fang", "gangxia fang", "junping fang", "tabei fang", "yuechi fang" and so on. Because of different terrains and home spaces, the whole town had "nine ridges", namely the "guanshang ridge", "xinfanggong ridge" and so on. 
There were also "thirteen lanes", namely, east lane, west lane and so on.

Fangs, ridges and the lanes were crisscrossed but could connect to the five-mile long street which was the main street in the town. There were cross streets, bridge streets close to the river on the west side of the five-mile long street. The businesses were prosperous on both sides of the street, through oral tradition, the "nine ridges and thirteen lanes, grass cloth shop everywhere". The wharf was divided into three parts, including "baima chau", "zhongzhou chau" and "shangsha chau" in order to dye and dry the grass cloth. The whole town was in good order at that time.

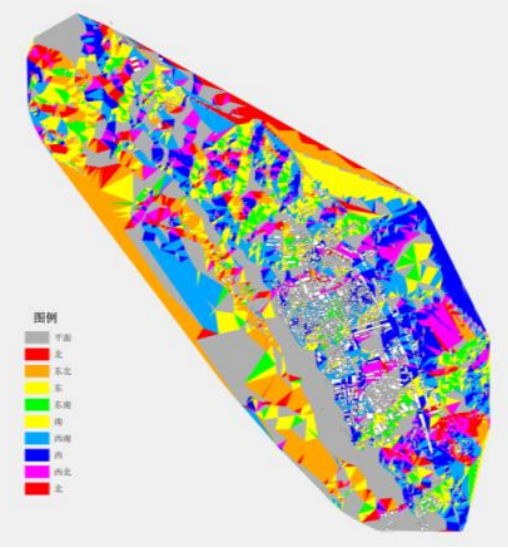

Figure 2. The aspect analysis for interpretation of the historical information

\subsection{With the potential, ancient street, cluster}

Tangyin town is located in hilly terrain between the plain area of Fuzhou city and Wuyi and Yunshan mountain. The construction followed the topography and the ancient street was parallel to the contour lines. The lanes were extended to the riverside from the ancient street as the grass cloth production as based on the river. In order to facilitate day lighting and ventilation, buildings were constructed to adapt to the terrain from low to high forming a progressive type layout.

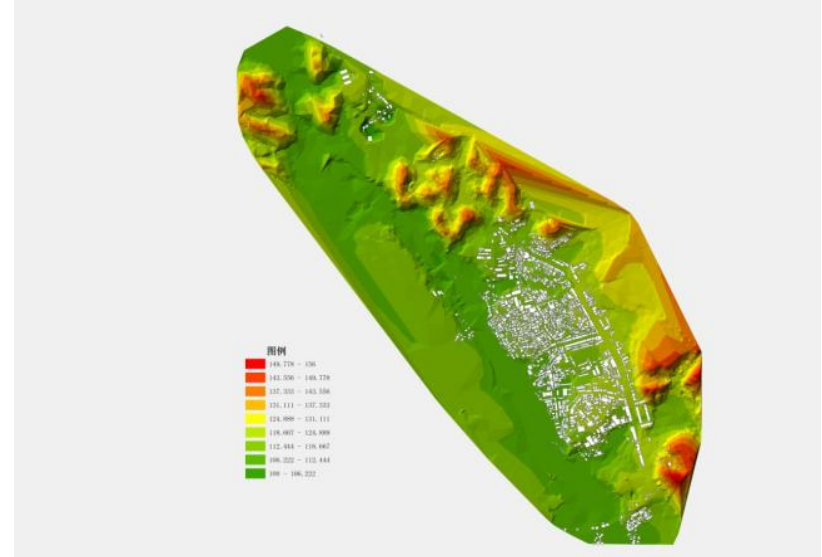

Figure 3. The elevation analysis for the settlement layout

\section{COURTYARD-SHAPE}

\subsection{Patio}

According to the day lighting and drainage differences, the courtyards can be divided into four types ${ }^{[3]}$, including waterform patio courtyard, soil -form courtyards, sunshade local-style courtyard and cellar well local-style courtyard. The scale and proportion of the patio depends on the size and shape of buildings.

The vernacular dwellings in Tangyin can be divided into three types. One is the inner- patio courtyard, one is the patio yard courtyard, and the third one is the half patio courtyard. The patio yard is larger than the ordinary patio and with little decoration. The patio which is located on two flanks stick on the wall called the half patio, commonly known as "the tiger's eye patio".

\subsection{Layout $^{[4]}$}

The historical and cultural heritage in Tangyin including residential buildings, the ancestral temples, branch of the ancestral temples, the public's ancestral temples and so on. The residential buildings are the main one. There are two main branches of the surname in Tangyin. One is $\mathrm{Wu}$, the other one is Luo. Therefore, the ancestral temples of $\mathrm{Wu}$ and Luo are the biggest and most ancient temples in town. The residential buildings almost surround the ancestral temple.

The traditional buildings in Tangyin are mainly influenced by the traditional culture of the middle area of Jiangxi province. Limited by the space, the traditional courtyards are overall outlined in irregular shapes. In contrast, the buildings in the main axis of the courtyard are much more structured. The number of patios in the main axis represent the numbers of steps. There are four types of traditional courtyards in Tangyin, namely one-step courtyard, two-step courtyard, three-step courtyard and the courtyard without patio.

\subsubsection{The courtyard without patio}

Mainly includes the "line-styled" and the "L-styled". These kinds of courtyards mostly layout along the street and have their own dressing rooms.

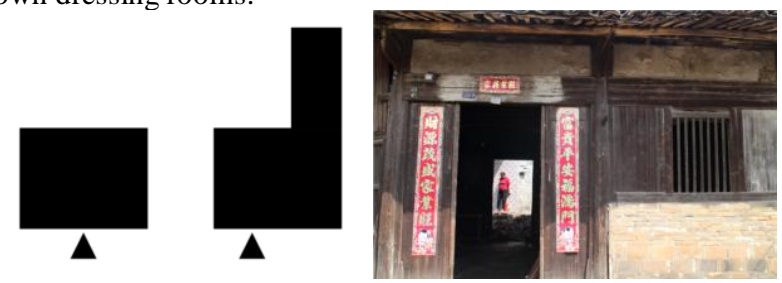

Figure 4. The mode chart of "line-styled" and the "L-styled" courtyards

\subsubsection{The one-step courtyard}

According to with or without the opposite hall, the direction of the courtyard entrance, with or without the patio yard, it is divided into three types as following.

Firstly, it is the "patio + hall" type. The entrance to the courtyard is generally located on the right side of the patio. The patio of this kind is mainly the "half patio".

Secondly, it is the "opposite hall + patio + hall" type. There are three entrance forms. The first entrance form is on the left side of the opposite hall, which is generally located in the lane. The second entrance form is on the right side of the opposite hall. The third form is in the middle of the opposite hall, which has screen in the opposite side of the courtyard entrance.

Thirdly, it is the "patio yard + opposite hall+ patio + hall" type. The entrance is generally located deviating from the central axis. 

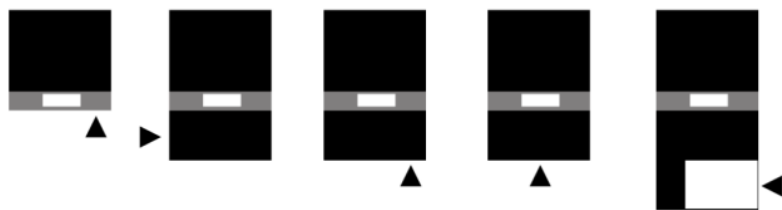

Figure 5. The mode chart of the one-step courtyards

\subsubsection{The two-step courtyard}

According to the with or without the opposite hall, the direction of the courtyard entrance, with or without the patio yard and with or without auxiliary axis, it is divided into two types as following.

Firstly, it is the "opposite hall + patio + hall+ patio + hall " type. There are two entrance forms. One is in the middle of the opposite hall, which has screen in the opposite side of the courtyard entrance. One is on the left side of the opposite hall. Secondly, it is the "main axis + auxiliary axis". This type adds a column auxiliary compound (generally on the left side) on the basis of the first type. According to with or without the patio yard and its position, it is divided into three kinds of circumstances. One has a patio yard before and after the courtyard. One is without patio yard. One is located in auxiliary axis.
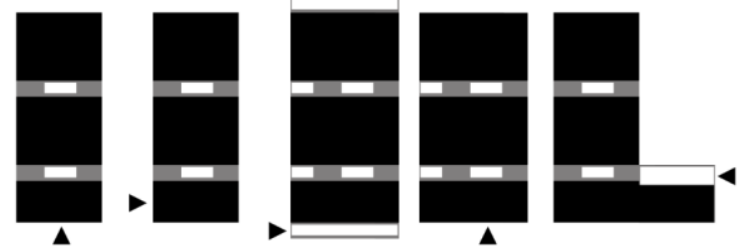

Figure 6 . The mode chart of the two-step courtyards

\subsubsection{The three-step courtyard}

Besides the main axis, this kind of courtyard always has auxiliary axis on each side, which is called “川" style in common.According to the census, the three-step courtyard always has patio yard. Based on the position and size of the patio yard, it can be divided into three types.

Firstly, it is the "small patio yard" type. The size of the patio yard is long and narrow.Secondly, it is the "big patio yard" type. The width of the patio yard is wider than the width of the hall. Thirdly, it is the "multi-patio yards" type. There is only one entity right now, named "huge courtyard of $\mathrm{Wu}$ family" which is the biggest vernacular dwelling in Tangyin.

The buildings in the main axis are arranged in good order. The hall is the in the most important position. The buildings in auxiliary axis are arranged according to the space residue.
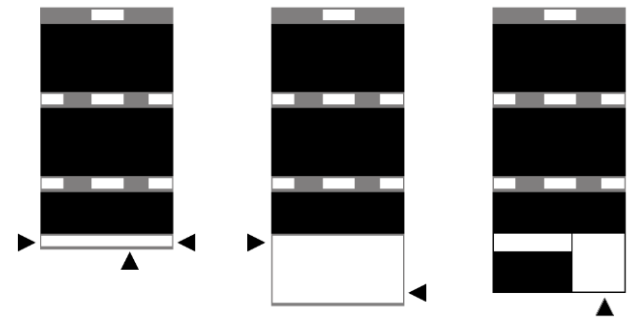

Figure 6 . The mode chart of the three-step courtyards

\subsection{Ventilation, lighting and drainage}

The ventilation is based on the unit of "door+ window+ patio+ door", patio as the core. Narrow high patios pull the wind to make good ventilation. The hall faces the patio and is completely open and the daylight is visible.

The main function of the patio is to meet the interior lighting, ventilation and drainage which and is not a space for human activities. The aspect ratio of the patio is about 2 to 1 , its width and normal eaves height ratio is approximately ranged from 1:1 to $2: 1$. Such proportion of the patio is beneficial to form stable negative pressure zones to achieve good ventilation affection.

The traditional buildings in the southern part of China are almost wooden frame structures with the brick wall filling in between the structure. At that time, glass was not commonly used in construction. In order to defend against criminal and fire, it was unfavourable to have large open windows on the outside wall. Therefore the window facing the patio was developed. Introverted daylight is an important feature of the traditional patio buildings.

Drainage was constructed under the patio to ensure rain water did not enter from the roof.

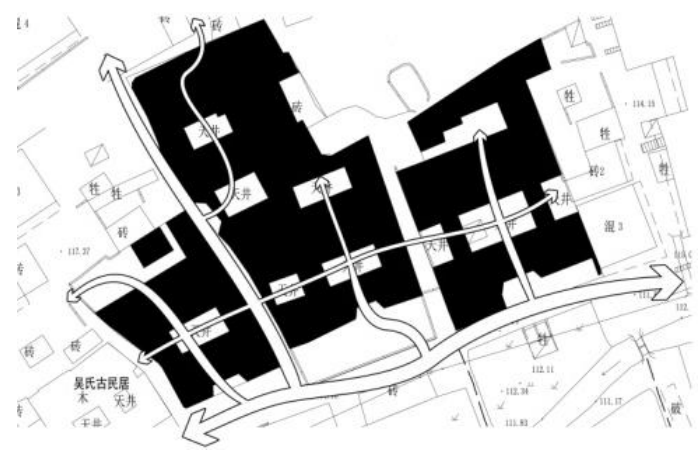

Figure 7. The ventilation mode chart of the cluster

\section{THE ARCHITECTURE CHARACTORS OF THE HALL}

\subsection{Lifting beams and tenon through structure}

The structure of the legacy ancient dwellings is almost tenon through the Qing dynasty. In order to get more space, the columns was reduced and transferred to be lifting beams structure.

The backbone beam of hall is moon shaped with beautiful carves and paintings.
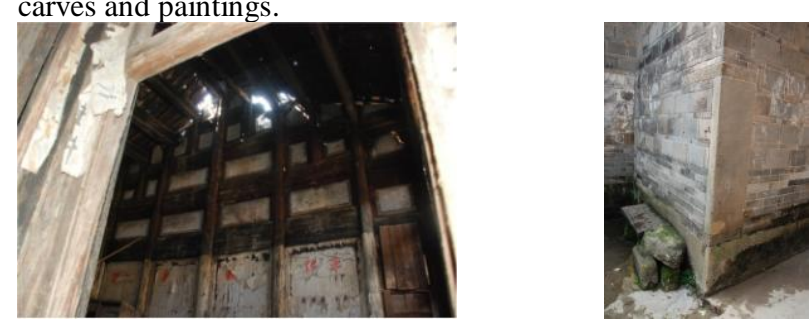

Figure 8. Pictures of the building structure and the rowlock wall

\subsection{Rowlock wall, horse-head wall ${ }^{[6]}$}

The outer wall of the traditional buildings is a rowlock wall, a sleep a pipe laying, filling the hollow part with grass mud to improve thermal performance. The rowlock wall is a kind of non-homogeneous hollow wall masonry. The robustness is poorer than a solid wall of bricks or stones. So for the important parts of the wall, such as doors and windows on both sides, and 
the junction of vertical and horizontal wall, the following $3 \sim 4$ skin brick wall below the indoor and outdoor floor and the part of floor slab under concentrated load are built by laying bricks or stones one on top of another.

\subsection{Gates and windows}

\subsubsection{The courtyard gate, the front door}

The gate types of Jiangxi traditional dwellings are abundant. In addition to the "line-type" door, the splayed door, arch door and the door with post and column are also used a lot. Except for the red stone, the pea green stone is also commonly used for the framework material of the gate. The courtyard gate generally uses stone frame with pillow stones and threshold beneath.
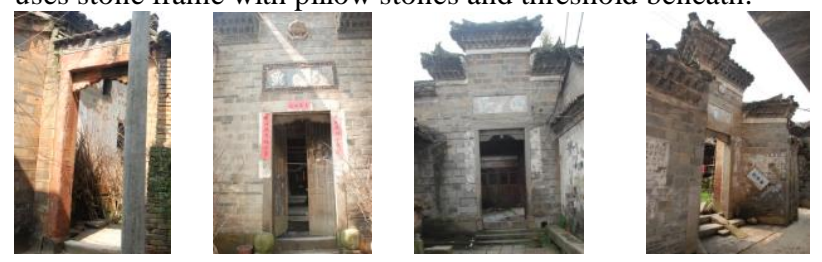

Figure 9. Different patterns of gates' pictures

\subsubsection{The window}

Generally the exterior wall of the opposite hall will set flower window carved flowers and plants, trees, birds and animals or other beautiful patterns. The decoration function is intensified and the practical function, such as ventilation or day lighting, is weak.

The wing buildings generally adopt window mullions with a variant type of "one horse and three arrows" windows which set the transverse wood at the upper middle and lower part of the window mullion. There are often more than three horizontal transverse wood pieces, which is the character of Tangyin.

\subsection{The floor of the hall}

The material of the floor in Jiangxi province generally is granite. Lime, yellow, tung oil, rice milk paved with a variety of materials are also used. While in Tangyin, the floor materials are mostly square bricks paved in inclined pattern. On the edge of the floor, granite slabs are also used.

\subsection{The furniture in the hall}

In the middle of the hall, opposite the gate, adjoining the wall, is always a warped head desk which is a necessary piece of furniture in the hall. But it scarcely can be seen currently. Instead a new desk for sacrifice, normally simple pattern is used. The difference from furnishings on the normal warped head desk is the china scarcely can be seen. In the middle of the desk, they still place bronze tripods and incense burners, which are heavier and bigger vessels (stone carvings and jade carvings are less layout). The hall generally has sacrifice function, so the memorial tablets or niches are always placed in the middle of the desk.

\section{CONCLUSIONS}

\subsection{The influence factors of the construction}

Besides the climatic factor in the middle area of Jiangxi province, there are still some factors for the settlement's formation as followings.

5.1.1 The rise of the merchants in Jiangxi province

Thanks to advantageous properties and natural resources of Tangyin together with the harsh policy on marine trade, the development of inland trade such as the business of Tangyin had been promoted. The convenience of the river transportation and the knave movement further improves the development of local businesses.

The prosperity of the local economy in Tangyin led to the expansion of investment. After attaining wealth, the businessmen purchased fields and built dwellings and ancestral temples. All of these led to the development of urbanism.

\subsubsection{The concept of fengshui}

According to the fengshui theory, predecessors constructed in their selected address point successively. The structure of the settlement is also based on this theory that different streets and lanes criss-cross, and behindthe settlements are small hills and mountains which are also part of fengshui. Thirdly, the residents built dwellings with well implied meaning and the decoration of their houses are also with some good meanings for wealth, health and so on.

\subsubsection{The application of local materials}

The construction materials are mainly local ones. The bricks are made from the soil which can be obtained from the surrounding hills. The structure of the wall uses bamboo, which is one of the main plants in Tangyin. Columns and other wood structural members are made of Cedar wood, which is good for keeping away moisture.

\subsection{The features of the construction logic}

Above all, as the figure shows in the following, based on local culture, such as feng shui and ancestral ideas, the original site selection has been complete. Then the natural and artificial surroundings appear gradually. The scale of land use and the scale of population are booming. The structure of the settlement, such as the streets and lanes, the residential clusters are gradually stabilizing. The development of the local economy promoted cultural exchange, which affects the construction features, no matter the whole layout or the buildings. Together with the local building technology, the construction activities in Tangyin appear with some differences from other areas of Jiangsu province,. The materials selected from Tangyin itself, but some beams or columns need to be much bigger, so wood is transfer from Fujian province. The decoration is mainly based on regional methods, but variation also exists. 


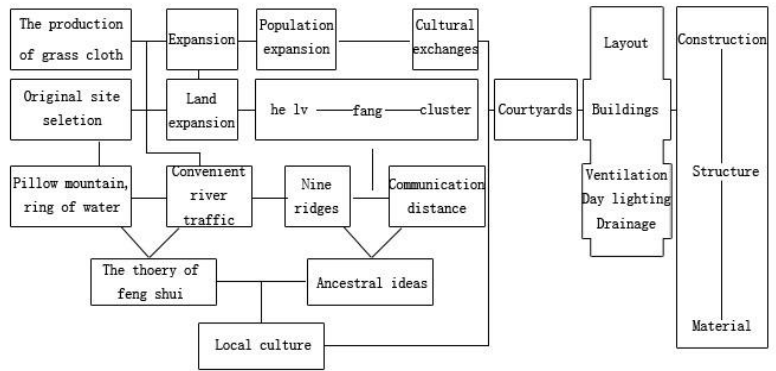

Figure 10. The chart of construction logic in Tangyin

\section{REFERENCES}

[1] Xu huailin,2000. The interaction changes between economic development and the ecological environment in the history of Jiangxi. Agricultural Archaeology, pp.110-124

[2] Li Jie,2013.The Process and Development of Vernacular Dwelling in Jiangxi Province. Architectural Culture,pp.165-167

[3] Housing and urban-rural development of the People's Republic of China,2014."The China traditional local-style dwelling houses type complete works". Beijing: China Building Industry Press.

[4] Pan ying,2009. The planar pattern interpretation of Jiangxi traditional local-style dwelling houses,pp.197-199

[5] Huang hao,1996. The degenerate and disappear in the traditional local-style dwelling houses. The traditional local-style dwelling houses and culture of China (7) -the seventh conference proceedings of Chinese local-style dwelling houses, pp.19-26. Agricultural Archaeology, pp.197-199

[6]Shi ying,2009. The analysis of the outer walls of the traditional dwellings in Jiangxi. Agricultural Archaeology, pp.200-202

\section{APPENDIX}

"He lu" is a traditional residential unit. "Fang" is a traditional residential unit. 